\title{
Energy Loss in Pulse Detonation Engine due to Fuel Viscosity
}

\author{
Weipeng Hu, ${ }^{1,2,3}$ Zichen Deng, ${ }^{1,2}$ and Gongnan Xie ${ }^{4}$ \\ ${ }^{1}$ School of Mechanics, Civil Engineering and Architecture, Northwestern Polytechnical University, Xian, Shaanxi 710072, China \\ ${ }^{2}$ State Key Laboratory of Structural Analysis of Industrial Equipment, Dalian University of Technology, Dalian, Liaoning 116023, China \\ ${ }^{3}$ Guangxi Key Laboratory of Disaster Prevention and Structural Safety, Guangxi University, Nanning, Guangxi 530004, China \\ ${ }^{4}$ School of Mechanical Engineering, Northwestern Polytechnical University, Xian, Shaanxi 710072, China
}

Correspondence should be addressed to Weipeng Hu; wphu@nwpu.edu.cn

Received 7 April 2014; Accepted 5 May 2014; Published 29 May 2014

Academic Editor: Zhijun Zhang

Copyright (C) 2014 Weipeng Hu et al. This is an open access article distributed under the Creative Commons Attribution License, which permits unrestricted use, distribution, and reproduction in any medium, provided the original work is properly cited.

Fluid viscosity is a significant factor resulting in the energy loss in most fluid dynamical systems. To analyze the energy loss in the pulse detonation engine (PDE) due to the viscosity of the fuel, the energy loss in the Burgers model excited by periodic impulses is investigated based on the generalized multisymplectic method in this paper. Firstly, the single detonation energy is simplified as an impulse; thus the complex detonation process is simplified. And then, the symmetry of the Burgers model excited by periodic impulses is studied in the generalized multisymplectic framework and the energy loss expression is obtained. Finally, the energy loss in the Burgers model is investigated numerically. The results in this paper can be used to explain the difference between the theoretical performance and the experimental performance of the PDE partly. In addition, the analytical approach of this paper can be extended to the analysis of the energy loss in other fluid dynamic systems due to the fluid viscosity.

\section{Introduction}

Improving the efficiency of the pulse detonation engine (PDE) is an everlasting objective for the researchers in the associated fields. Generally, the efficiency of the PDE is determined by the energy loss due to the viscosity of the fuel, the heat dissipation of the structure, and other factors, in which the energy loss due to the viscosity of the fuel is a major factor.

In the current research findings, the effects on the efficiency of the PDE from the structure parameters of PDE, the mass fraction of the fuels, and the frequency of the detonation have been reported. For example, Bratkovich and Bussing [1] presented a PDE performance model, which considered inlet, mixer, combustor, and nozzle contributions to PDE performance attributes, referring to the ideal PDE model presented by Endo and Fujiwara [2]. Radulescu and Hanson [3] presented a study to address the influence of convective heat losses on the flow field and performance of PDE, considering various working conditions; Tangirala and Dean [4] proposed a performance estimation model for the PDE with exit nozzle; Glaser [5] studied the effect on the efficiency of the PDE from the structure parameters of PDE in laboratory; and Brophy and Hanson [6] investigated the fuel distribution effects on pulse detonation engine operation and performance. Recently, Yan and colleagues [7-10] studied the fuel filled length, the frequency of the detonation, and the structure parameters of PDE effects on the performance of the PDE.

For the limitation of the measuring means, the energy loss due to the viscosity of the fuel in the PDE has not been reported in current literatures, while the energy losses due to viscosity of the fluid in some fluid dynamic models have been investigated in many literatures [11-15]. The research results implied that the energy losses due to viscosity could not be neglected in the majority of fluid models. Thus, the energy loss due to the viscosity of the fuel in the PDE is presented by studying the energy loss due to the viscosity of the fluid in Burgers model excited by periodic impulses. Focusing on the energy loss due to the viscosity of the fluid in the PDE, a Burgers model excited by periodic impulses is introduced to describe the flow field in the PDE firstly; and then based on the generalized multisymplectic theory [1618], the expression of the energy loss due to the viscosity of 
the fluid is derived from the first-order symmetric form of the Burgers model; finally, the numerical results of the energy loss are reported.

\section{Energy Loss in PDE Model due to Fuel Viscosity}

The practical model of the PDE in laboratory is a slender tube; thus the practical flow field in the detonation tube is threedimensional. Ignoring the effect of the boundary layer, the flow field on arbitrary cross section in the detonation tube is homogeneous approximately. With this assumption, the three-dimensional flow field in the detonation tube can be simplified to one-dimensional flow field.

In addition, the purpose of this paper is to investigate the energy loss due to the viscosity of the fuel; thus the effect of the chemical reactions among the components of the fuel can be represented by a series of periodic impulses. Then the flow fields in the detonation tube can be expressed by the following Burgers model:

$$
\partial_{t} u+u \partial_{x} u-v \partial_{x x} u=f(t, x) \quad x \in[0, L]
$$

where $v$ is the kinematics viscosity of the fuel.

$$
f(t, x)=A \sum_{n=0}^{N} \Psi \delta\left(t+n T, V t-V \varphi_{0}-n V T+x_{0}\right)
$$
$\left(n=0,1, \ldots, N, N=\left[\left(t-\varphi_{0}\right) / T\right],[\cdot]\right.$ is the symbol of the rounding operation) are sequence of periodic impulses that describes the effect of the periodic detonations resulting from the chemical reactions among the components of the fuel. $t+n T$ is the moment of the impulse and $V t-V \varphi_{0}-$ $n V T+x_{0}$ is the position of the impulse that satisfies $0 \leq$ $V t-V \varphi_{0}-n V T+x_{0} \leq L ; A$ is a constant that denotes the amplitude of periodic detonation wave. $\Psi$ is a switch function: $\Psi=\left\{\begin{array}{cc}0 & 0 \leq t<\varphi_{0} \\ 1 & t \geq \varphi_{0}\end{array} . L\right.$ is the length of the detonation tube. $T$ is the period of the detonation. $V$ is the propagation speed of the detonation wave. $x_{0}$ is the position where the first detonation wave appears. $\varphi_{0}$ is the time lag between burning the fuel and appearing of the first detonation wave.

Equation (1) can be used to describe the flow filed of the transient state as well as the steady state in the detonation tube. The symmetric form of (1) can be obtained referring to the results of [17].

Introducing canonical momenta $\partial_{x} p=u, \partial_{x} q=-(1 / 2)$ $\partial_{t} u$, and defining state variable $\mathbf{z}=(p, u, q)^{T}$, (1) becomes the first-order partial differential equations

$$
\begin{aligned}
& {\left[\begin{array}{ccc}
0 & \frac{1}{2} & 0 \\
-\frac{1}{2} & 0 & 0 \\
0 & 0 & 0
\end{array}\right] \partial_{t} \mathbf{z}+\left(\left[\begin{array}{ccc}
0 & 0 & 1 \\
0 & 0 & 0 \\
-1 & 0 & 0
\end{array}\right]+\left[\begin{array}{lll}
0 & 0 & 0 \\
0 & v & 0 \\
0 & 0 & 0
\end{array}\right]\right) \partial_{x} \mathbf{z}} \\
& =\nabla_{\mathbf{z}} S(\mathbf{z})+\mathbf{F}(t, x)
\end{aligned}
$$

where $S(\mathbf{z})=-u q+(1 / 6) u^{3}$ is the Hamiltonian function and the external excitation is

$$
\begin{aligned}
\mathbf{F}(t, x) & \\
& =\left(0, \int f(t, x) \mathrm{d} x, 0\right)^{\mathrm{T}} \\
& =\left(0, \int A \sum_{n=0}^{N} \Psi \delta\left(t+n T, V t-V \varphi_{0}-n V T+x_{0}\right) \mathrm{d} x, 0\right)^{\mathrm{T}} \\
& =\left(0, A L \sum_{n=0}^{N} \Psi \mathcal{E}\left(t+n T, V t-V \varphi_{0}-n V T+x_{0}\right), 0\right)^{\mathrm{T}}
\end{aligned}
$$

here $\varepsilon\left(t+n T, V t-V \varphi_{0}-n V T+x_{0}\right)$ is the step function.

For simplicity, skew-symmetric matrices $\mathbf{M}, \mathbf{K}$, and symmetric matrix $\widehat{\mathbf{K}}$ are defined as

$$
\begin{gathered}
\mathbf{M}=\left[\begin{array}{ccc}
0 & \frac{1}{2} & 0 \\
-\frac{1}{2} & 0 & 0 \\
0 & 0 & 0
\end{array}\right], \quad \mathbf{K}=\left[\begin{array}{ccc}
0 & 0 & 1 \\
0 & 0 & 0 \\
-1 & 0 & 0
\end{array}\right], \\
\widehat{\mathbf{K}}=\left[\begin{array}{lll}
0 & 0 & 0 \\
0 & v & 0 \\
0 & 0 & 0
\end{array}\right] .
\end{gathered}
$$

Obviously, if $v=0$ and $\mathbf{F}(t, x)=\mathbf{0}$, the symmetric form (2) is a multisymplectic form $[19,20]$, which satisfies the multisymplectic conservation law and the local energy conservation law as well as the local momentum conservation law.

Actually, the kinematic viscosity coefficient of the fuel $v$ in the PDE is a positive real number, which makes the symmetric form (2) generalized multisymplectic [17] with external excitation. Following the outline of [17], the local energy error of the symmetric form (2) can be obtained [17]:

$$
\begin{aligned}
\Delta_{l}=\partial_{t}\left[A L \sum_{n=0}^{N} \Psi \varepsilon\left(t+n T, V t-V \varphi_{0}-n V T+x_{0}\right)\right. \\
\left.+S(\mathbf{z})-\frac{1}{2} \mathbf{z}^{\mathrm{T}}(\mathbf{K}+\widehat{\mathbf{K}}) \partial_{x} \mathbf{z}\right]+\frac{1}{2} \partial_{x} \mathbf{z}^{\mathrm{T}}(\mathbf{K}+\widehat{\mathbf{K}}) \partial_{t} \mathbf{z} \\
=A L(1+V) \sum_{n=0}^{N} \Psi \delta\left(t+n T, V t-V \varphi_{0}-n V T+x_{0}\right) \\
+\frac{\nu}{2} \partial_{x} u \partial_{t} u-\frac{\nu}{2} \partial_{t}\left(u \partial_{x} u\right) \\
=A L(1+V) \sum_{n=0}^{N} \Psi \delta\left(t+n T, V t-V \varphi_{0}-n V T+x_{0}\right) \\
\quad-\frac{\nu}{2} u \partial_{t x} u=\Delta_{l e}-\Delta_{l v},
\end{aligned}
$$


where the local energy error contains two parts: one is the local energy increment $\Delta_{l e}=A L(1+V) \sum_{n=0}^{N} \Psi \delta(t+n T, V t-$ $\left.V \varphi_{0}-n V T+x_{0}\right)$ resulting from the impulse excitation; another part is the local energy loss $\Delta_{l v}=(v / 2) u \partial_{t x} u$ due to the fluid viscosity.

Superficially, the local energy loss $\Delta_{l v}$ has nothing to do with all the detonation parameters $\left(L, V, T, x_{0}, \varphi_{0}\right.$, and $\left.A\right)$, but actually the effect of the detonation is included in the distribution of the flow field $u$.

From the local energy loss (3), it is easy to get the global energy error;

$$
\begin{aligned}
& \Delta_{g}= \int_{0}^{L} \Delta_{l} \mathrm{~d} x \\
&=\int_{0}^{L}\left[A L(1+V) \sum_{n=0}^{N} \Psi \delta\left(t+n T, V t-V \varphi_{0}-n V T+x_{0}\right)\right. \\
&\left.\quad-\frac{v}{2} u \partial_{t x} u\right] \mathrm{d} x .
\end{aligned}
$$

In this paper, we consider that the components of the fuel are mixed uniformly, which means that the kinematic viscosity coefficient of the fuel $\nu$ in the PDE is independent of time and position; thus, the global energy error is

$$
\begin{aligned}
\Delta_{g}= & \int_{0}^{L} A L \sum_{n=0}^{N} \Psi(1+V) \delta\left(t+n T, V t-V \varphi_{0}-n V T+x_{0}\right) \mathrm{d} x \\
& -\frac{v}{2} \int_{0}^{L} u \partial_{t x} u \mathrm{~d} x \\
= & A L^{2}(1+V) \sum_{n=0}^{N} \Psi \varepsilon\left(t+n T, V t-V \varphi_{0}-n V T+x_{0}\right) \\
& -\frac{\nu}{2} \int_{0}^{L} u \partial_{t x} u \mathrm{~d} x \\
= & \Delta_{g e}-\Delta_{g v} ;
\end{aligned}
$$

similarly, the global energy error contains two parts: one is the global energy increment $\Delta_{g e}=A L^{2}(1+V) \sum_{n=0}^{N} \Psi \varepsilon(t+$ $\left.n T, V t-V \varphi_{0}-n V T+x_{0}\right)$ resulting from the impulse excitation; another part is the global energy loss $\Delta_{g \nu}=(\nu / 2) \int_{0}^{L} u \partial_{t x} u \mathrm{~d} x$ due to the fluid viscosity.

\section{Generalized Multisymplectic Scheme}

To investigate the energy loss in PDE by generalized multisymplectic method $[16,17]$, the generalized multisymplectic scheme for the symmetric form (2) must be constructed firstly.

Referring to [17], introduce a uniform grid $\left\{\left(t_{i}, x_{j}\right)\right\}$ in $R^{2}$ with time step size $\Delta t$ in the $t$-direction and spatial step size $\Delta x$ in the $x$-direction. The approximate value of $\mathbf{z}(t, x)$ at the point $\left(t_{i}, x_{j}\right)$ is denoted by $\mathbf{z}_{j}^{i}$.
Using the Preissman box discrete method, the discrete form of the symmetric form (2) can be obtained:

$$
\begin{aligned}
& {\left[\begin{array}{ccc}
0 & \frac{1}{2} & 0 \\
-\frac{1}{2} & 0 & 0 \\
0 & 0 & 0
\end{array}\right] \delta_{t}^{+} \mathbf{z}_{j+1 / 2}^{i}+\left[\begin{array}{ccc}
0 & 0 & 1 \\
0 & v & 0 \\
-1 & 0 & 0
\end{array}\right] \delta_{x}^{+} \mathbf{z}_{j}^{i+1 / 2}} \\
& =\nabla_{\mathbf{z}} S\left(\mathbf{z}_{j+1 / 2}^{i+1 / 2}\right)+\mathbf{F}(i, j),
\end{aligned}
$$

where $\delta_{t}^{+}$is the forward differences to $\partial_{t} \mathbf{z}$ and $\delta_{x}^{+}$is the forward differences to $\partial_{x} \mathbf{z}, \mathbf{z}_{j+1 / 2}^{i}=\left(\mathbf{z}_{j+1}^{i}+\mathbf{z}_{j}^{i}\right) / 2, \mathbf{z}_{j}^{i+1 / 2}=$ $\left(\mathbf{z}_{j}^{i+1}+\mathbf{z}_{j}^{i 1}\right) / 2$, and $\mathbf{z}_{j+1 / 2}^{i+1 / 2}=\left(\mathbf{z}_{j+1}^{i+1}+\mathbf{z}_{j+1}^{i}+\mathbf{z}_{j}^{i+1}+\mathbf{z}_{j}^{i}\right) / 4$.

It has been proven that the discrete form (8) is generalized multisymplectic with structure-preserving property if the kinematic viscosity coefficient of the fuel $v$ is small enough [17].

The discrete form of the local energy error of the symmetric form (2) at the point $\left(t_{i}, x_{j}\right)$ is

$$
\begin{aligned}
\left(\Delta_{l}\right)_{j}^{i}= & A L(1+V) \sum_{n=0}^{N} \Psi \delta\left(i+n T, V i-V \varphi_{0}-n V T+x_{0}\right) \\
& -\frac{v}{2} u_{j+1 / 2}^{i+1 / 2} \delta_{t}^{+} \delta_{x}^{+} u_{j+1 / 2}^{i+1 / 2}=\left(\Delta_{l e}\right)_{j}^{i}-\left(\Delta_{l v}\right)_{j}^{i},
\end{aligned}
$$

where the local energy increment resulting from the impulse excitation is $\left(\Delta_{l e}\right)_{j}^{i}=A L(1+V) \sum_{n=0}^{N} \Psi \delta\left(i+n T, V i-V \varphi_{0}-\right.$ $\left.n V T+x_{0}\right)$ and the local energy loss due to the fluid viscosity is $\left(\Delta_{l v}\right)_{j}^{i}=(\nu / 2) u_{j+1 / 2}^{i+1 / 2} \delta_{t}^{+} \delta_{x}^{+} u_{j+1 / 2}^{i+1 / 2}$.

Similarly, the discrete form of the global energy error of the symmetric form (2) at the $t_{i}$ is

$$
\begin{aligned}
&\left(\Delta_{g}\right)^{i}= A L^{2}(1+V) \sum_{n=0}^{N} \Psi \varepsilon\left(i+n T, V i-V \varphi_{0}-n V T+x_{0}\right) \\
&-\frac{\nu \Delta x}{4}\left[u_{1 / 2}^{i+1 / 2} \delta_{t}^{+} \delta_{x}^{+} u_{1 / 2}^{i+1 / 2}+2 \sum_{j=1}^{(L / \Delta x)-1} u_{j+1 / 2}^{i+1 / 2} \delta_{t}^{+} \delta_{x}^{+} u_{j+1 / 2}^{i+1 / 2}\right. \\
&\left.+u_{(L / \Delta x)+1 / 2}^{i+1 / 2} \delta_{t}^{+} \delta_{x}^{+} u_{(L / \Delta x)+1 / 2}^{i+1 / 2}\right] \\
&=\left(\Delta_{g e}\right)^{i}-\left(\Delta_{g \nu}\right)^{i},
\end{aligned}
$$

where the global energy loss due to the fluid viscosity is $\left(\Delta_{g \nu}\right)^{i}=(\nu \Delta x / 4)\left[u_{1 / 2}^{i+1 / 2} \delta_{t}^{+} \delta_{x}^{+} u_{1 / 2}^{i+1 / 2}+\right.$ $\left.2 \sum_{j=1}^{(L / \Delta x)-1} u_{j+1 / 2}^{i+1 / 2} \delta_{t}^{+}\left(\delta_{x}^{+} u_{j+1 / 2}^{i+1 / 2}\right)+u_{(L / \Delta x)+1 / 2}^{i+1 / 2} \delta_{t}^{+} \delta_{x}^{+} u_{(L / \Delta x)+1 / 2}^{i+1 / 2}\right]$ (we select the trapezoid quadrature method to calculate the numerical integration $\left.\int_{0}^{L} u \partial_{t x} u \mathrm{~d} x\right)$ and the global energy increment resulting from the impulse excitation is $\left(\Delta_{g e}\right)^{i}=A L^{2}(1+V) \sum_{n=0}^{N} \Psi \varepsilon\left(i+n T, V i-V \varphi_{0}-n V T+x_{0}\right)$. 


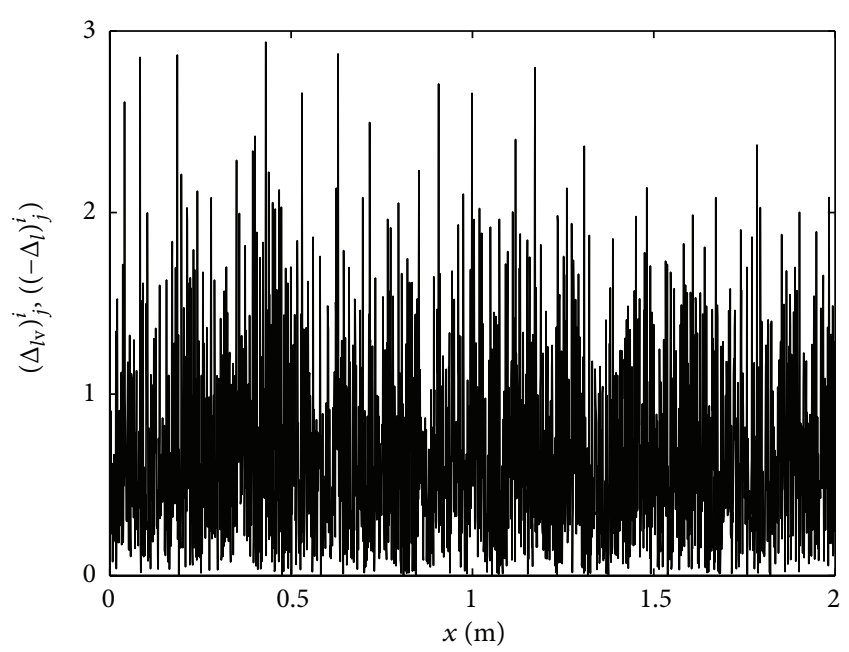

Figure 1: Local energy error and local energy loss due to the viscosity of the fuel on each grid at $t=5 \times 10^{-8} \mathrm{~s}$.

\section{Numerical Results}

In this section, some numerical results about the energy loss in PDE will be presented. In the numerical experiments, we let the step lengths be $\Delta t=5 \times 10^{-9} \mathrm{~s}$ and $\Delta x=0.01 \mathrm{~m}$, structure parameter of the PDE be $L=2 \mathrm{~m}$, the detonation parameters be $A=100 \mathrm{~m} \cdot \mathrm{s}^{-2}, V=2000 \mathrm{~m} \cdot \mathrm{s}^{-1}, x_{0}=0.2 \mathrm{~m}$, $\varphi_{0}=1 \times 10^{-7} \mathrm{~s}$, and $T=0.02 \mathrm{~s}$ (which implies that the detonation frequency is $50 \mathrm{~Hz}$ ) and the kinematic viscosity coefficient of the fuel $v=0.1$. The inset boundary condition considered in this paper is $u(t, 0)=100 \mathrm{~m} \cdot \mathrm{s}^{-1}$.

We simulate the flow field in the PDE by the structurepreserving scheme (8) and calculate the energy error on each grid. The numerical results of the local energy error and the local energy loss due to the viscosity of the fuel at different time are shown in Figures 1 and 2.

The local energy error is equal to the negative local energy loss due to the viscosity of the fuel on each grid at $t=5 \times 10^{-8} \mathrm{~s}$ $\left(\left(\Delta_{l}\right)_{j}^{i}=-\left(\Delta_{l v}\right)_{j}^{i}\right)$, and the local energy error on each grid is negative, see Figure 1, which implies that the local energy increment resulting from the impulse excitation on each grid $\left(\left(\Delta_{l e}\right)_{j}^{i}\right)$ is zero $(\Psi=0)$ at $t=5 \times 10^{-8} \mathrm{~s}$.

The local energy error, the local energy loss due to the viscosity of the fuel, and the local energy increment resulting from the impulse excitation increase quickly on the grids near the detonation wave at $t=4 \times 10^{-4} \mathrm{~s}$, see Figure 2 , which implies that the local energy loss due to the viscosity of the fuel increases when the speed of the fuel increases.

Based on the numerical results of the local energy error, the global energy error, the global energy increment resulting from the impulse excitation, and the global energy loss due to the fluid viscosity at each time step can be easily obtained. Figure 3 implies that the global energy loss due to the fluid viscosity can not be neglected in PDE. Neglecting the global energy loss due to the fluid viscosity will exaggerate the thrust of the PDE (here, the thrust is represented by the global energy increment) by $\left(\left(5.436924761525581 \times 10^{4}\right) /\left(8 \times 10^{5}\right)\right) \times$ $100 \% \approx 6.7962 \%$ at $t=5 \times 10^{-4} \mathrm{~s}$.
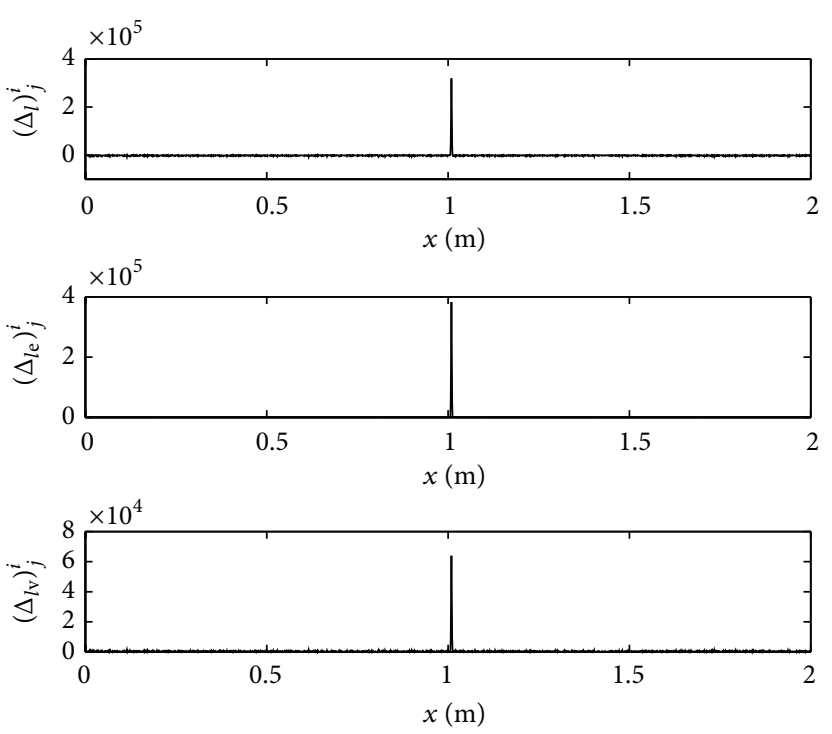

FIGURE 2: Local energy error, local energy increment resulting from the impulse excitation, and local energy loss due to the viscosity of the fuel on each grid at $t=4 \times 10^{-4} \mathrm{~s}$.
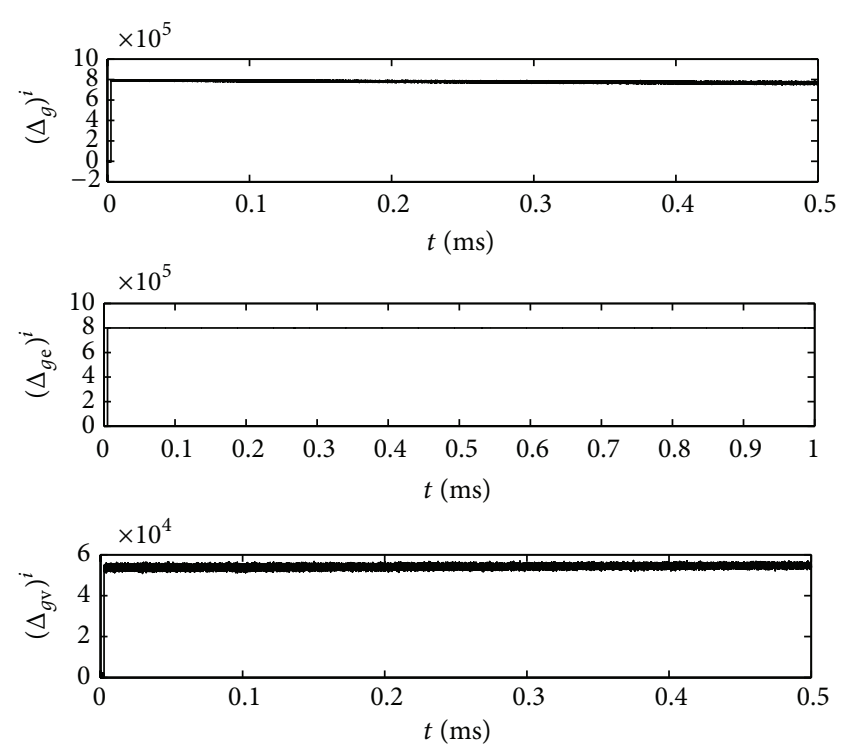

FIGURE 3: Global energy error, global energy increment resulting from the impulse excitation, and global energy loss due to the viscosity of the fuel on each grid at $t=4 \times 10^{-4} \mathrm{~s}$.

\section{Conclusion}

Energy loss in PDE due to the viscosity of the fuel is an important factor that affects the efficiency of PDE. In this paper, the energy loss due to the fuel viscosity is investigated numerically. A Burgers model excited by periodic impulses is established to describe the detonation process of PDE firstly. Then, the energy loss expressions are derived from the generalized multisymplectic form of the Burgers model. Finally, the numerical results of the energy loss in PDE are reported. From the results, it can be concluded that the energy loss 
due to the fuel viscosity accounts for $6.7962 \%$ of the energy increment resulting from the impulse excitation.

\section{Conflict of Interests}

The authors declare that there is no conflict of interests regarding the publication of this paper.

\section{Acknowledgments}

The research is supported by the National Natural Science Foundation of China (11372252 and 11372253), the Science Foundation of Aviation of China (2013ZB53020), the Fundamental Research Funds for the Central Universities (3102014JCQ01035), the Open Project of Guangxi Key Laboratory of Disaster Prevention and Structural Safety (2013ZDK04), and the Open Foundation of State Key Laboratory of Structural Analysis of Industrial Equipment (GZ1312).

\section{References}

[1] T. E. Bratkovich and T. R. A. Bussing, "A pulse detonation engine performance model," in Proceedings of the 31st AIAA/ ASME/SAE/ASEE Joint Propulsion Conference \& Exhibit, AIAA 95-3155, pp. 1-15, San Diego, Calif, USA.

[2] T. Endo and T. Fujiwara, "Analytical estimation of performance parameters of an ideal pulse detonation engine," Transactions of the Japan Society for Aeronautical and Space Sciences, vol. 45, no. 150, pp. 249-254, 2003.

[3] M. I. Radulescu and R. K. Hanson, "Effect of heat loss on pulsedetonation-engine flow fields and performance," Journal of Propulsion and Power, vol. 21, no. 2, pp. 274-285, 2005.

[4] V. E. Tangirala and A. J. Dean, "Performance estimations of a pulse detonation engine with exit nozzle," in Proceedings of the AIAA/ASME/SAE/ASEE 42nd Joint Propulsion Conference, pp. 4724-4739, Sacramento, Calif, USA, July 2006.

[5] A. J. Glaser, Performance and Environmental Impact Assessment of Pulse Detonation Based Engine Systems, University of Cincinnati, Cincinnati, Ohio, USA, 2007.

[6] C. M. Brophy and R. K. Hanson, "Fuel distribution effects on pulse detonation engine operation and performance," Journal of Propulsion and Power, vol. 22, no. 6, pp. 1155-1161, 2006.

[7] Y. Yan, W. Fan, K. Wang, and Y. Mu, "Experimental investigation of the effect of bell-shaped nozzles on the two-phase pulse detonation rocket engine performance," Combustion, Explosion and Shock Waves, vol. 47, no. 3, pp. 335-342, 2011.

[8] W. Fan, J. Li, Q. Li, and C. Yan, "Numerical investigation on multi-cycle operation of pulse detonation rocket engine," International Journal of Turbo and Jet Engines, vol. 25, no. 3, pp. 189195, 2008.

[9] Q. Zhang, C. Yan, W. Fan, and Q. Li, "Experimental investigation of effect of partial filling on the impulse of pulse detonation engine," Chinese Science Bulletin, vol. 52, no. 20, pp. 2859-2865, 2007.

[10] Q. Li, W. Fan, C.-J. Yan, C.-Q. Hu, and B. Ye, "Experimental investigation on performance of pulse detonation rocket engine model," Chinese Journal of Aeronautics, vol. 20, no. 1, pp. 9-14, 2007.

[11] C.-H. Yap, L. P. Dasi, and A. P. Yoganathan, "Dynamic hemodynamic energy loss in normal and stenosed aortic valves," Journal of Biomechanical Engineering, vol. 132, no. 2, 2010.
[12] K. Dusling, G. D. Moore, and D. Teaney, "Radiative energy loss and v2 spectra for viscous hydrodynamics," Physical Review C, vol. 81, no. 3, Article ID 034907, 2010.

[13] V. Fester, B. Mbiya, and P. Slatter, "Energy losses of nonNewtonian fluids in sudden pipe contractions," Chemical Engineering Journal, vol. 145, no. 1, pp. 57-63, 2008.

[14] T. Mai and T. Liebner, "Loss of energy dissipation capacity from the deadzone in linear and nonlinear viscous damping devices," Earthquake Engineering and Engineering Vibration, vol. 6, no. 1, pp. 11-20, 2007.

[15] S. Norris and G. Mallinson, "Volumetric methods for evaluating energy loss and heat transfer in cavity flows," International Journal for Numerical Methods in Fluids, vol. 54, no. 12, pp. 14071423, 2007.

[16] W. P. Hu, S. M. Han, and Z. C. Deng, "Analyzing dynamic response of non-homogeneous string fixed at both ends," International Journal of Non-Linear Mechanics, vol. 47, no. 10, pp. 1111-1115, 2012.

[17] W. Hu, Z. Deng, and S. Han, "An implicit difference scheme focusing on the local conservation properties for burgers equation," International Journal of Computational Methods, vol. 9, no. 2, Article ID 1240028, 2012.

[18] W. Hu, Z. Deng, S. Han, and W. Zhang, "Generalized multisymplectic integrators for a class of Hamiltonian nonlinear wave PDEs," Journal of Computational Physics, vol. 235, pp. 394-406, 2013.

[19] T. J. Bridges, "Multi-symplectic structures and wave propagation," Mathematical Proceedings of the Cambridge Philosophical Society, vol. 121, no. 1, pp. 147-190, 1997.

[20] T. J. Bridges and S. Reich, "Multi-symplectic integrators: numerical schemes for Hamiltonian PDEs that conserve symplecticity," Physics Letters, Section A: General, Atomic and Solid State Physics, vol. 284, no. 4-5, pp. 184-193, 2001. 


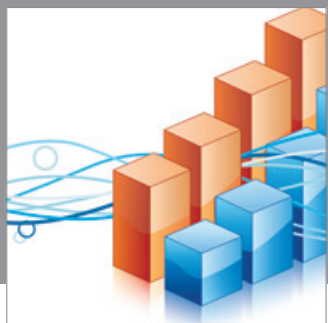

Advances in

Operations Research

mansans

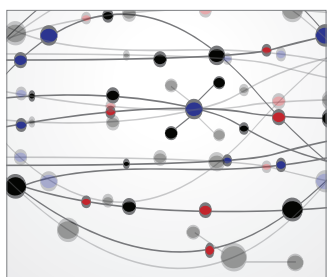

The Scientific World Journal
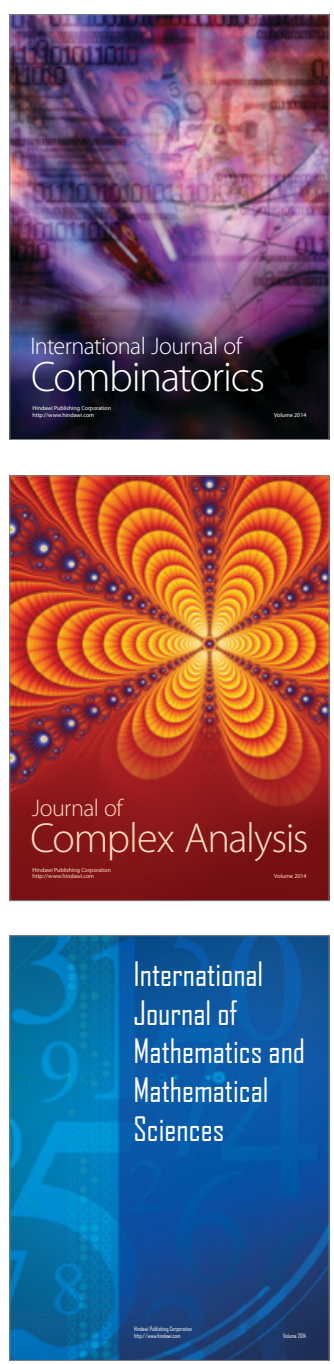
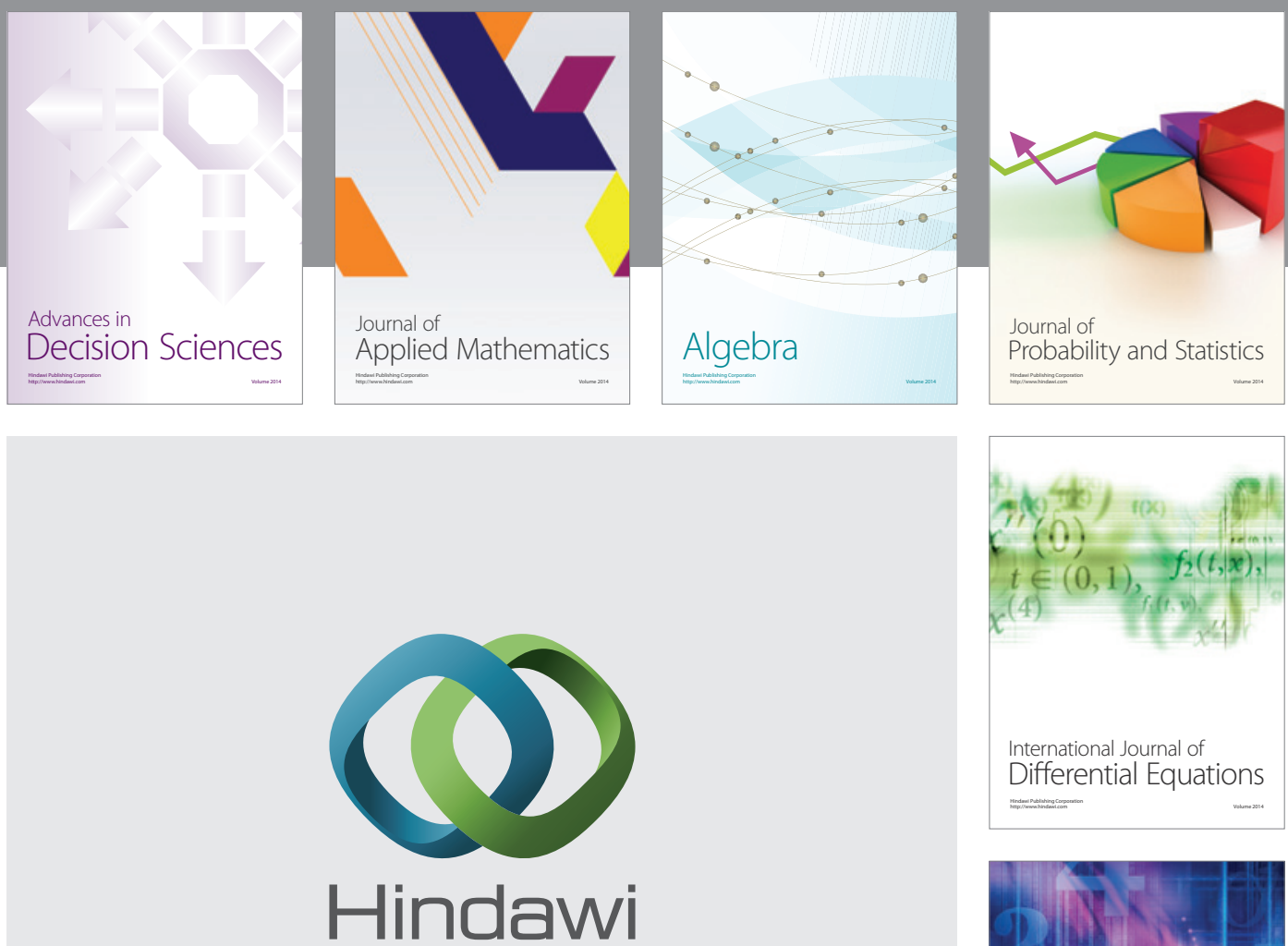

Submit your manuscripts at http://www.hindawi.com
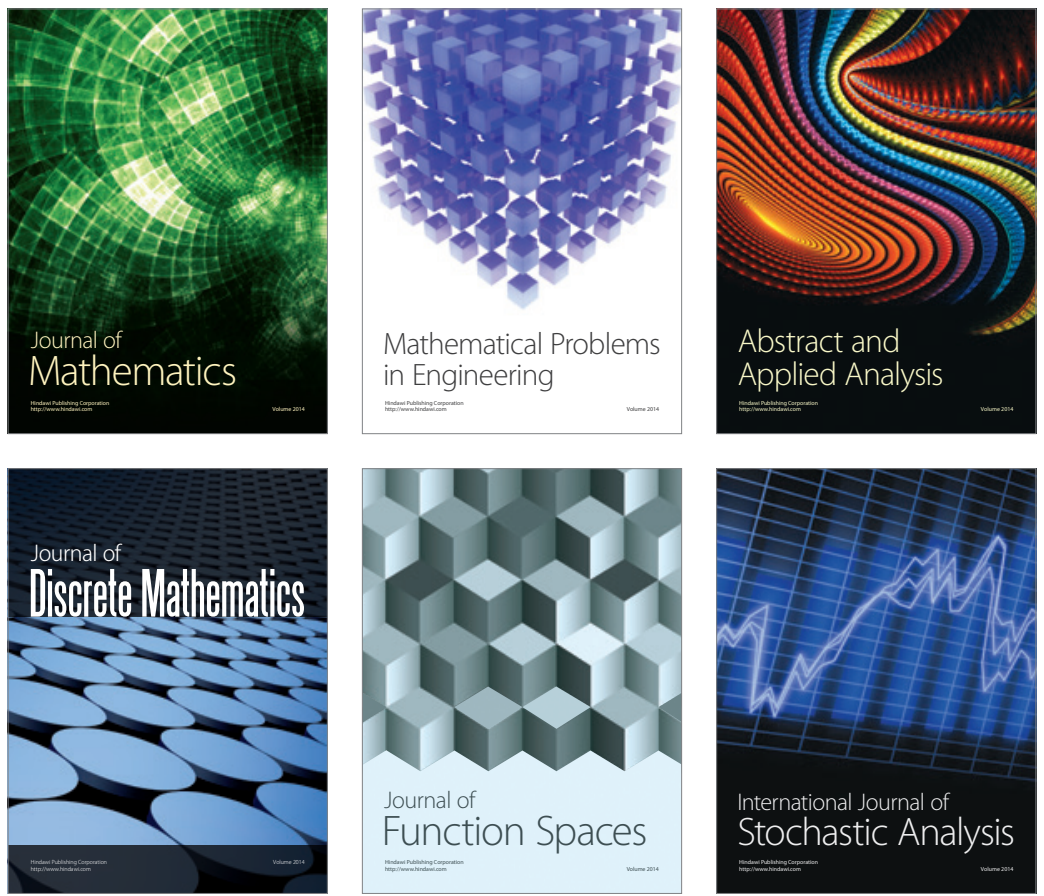

Journal of

Function Spaces

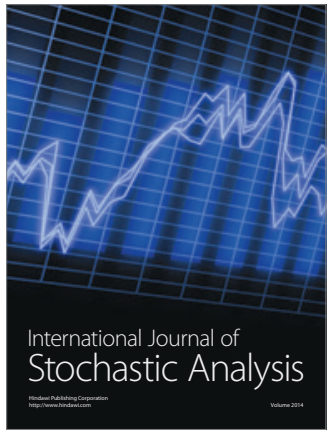

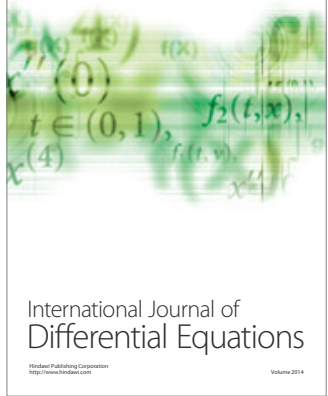
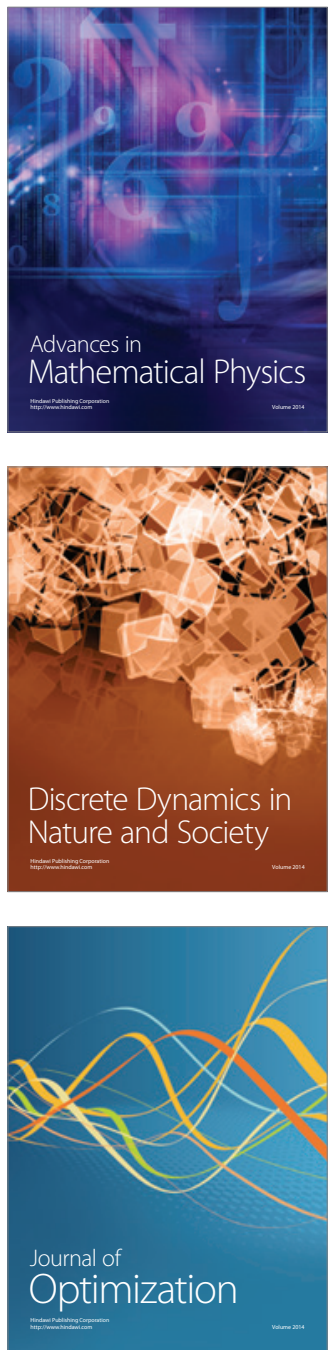